\title{
Histological classification of non-small cell lung cancer using Gabor filtering Minkowski functionals and Neighborhood Component Analysis on CT images.
}

Kentaro Yamagishi ( $\nabla$ yameken@med.u-toyama.ac.jp )

University of Toyama

Norihito Naruto

University of Toyama

Tatsuji Mizukami

University of Toyama

Junichi Saito

University of Toyama

kyo Noguchi

University of Toyama

\section{Research Article}

Keywords: non-small cell lung cancer, histology, computed tomography, machine learning, Minkowski functionals, Gabor filter, neighborhood component analysis

Posted Date: April 7th, 2021

DOI: https://doi.org/10.21203/rs.3.rs-400123/v1

License: (c) (i) This work is licensed under a Creative Commons Attribution 4.0 International License.

Read Full License 


\title{
Histological classification of non-small cell lung cancer using Gabor filtering
} Minkowski functionals and Neighborhood Component Analysis on CT images.

Kentaro Yamagishi ${ }^{1}{ }^{1 \star}$, Norihito Naruto ${ }^{1}$, Tatsuji Mizukami ${ }^{1}$, Junichi Saito ${ }^{1} \&$ Kyo Noguchi ${ }^{1}$

${ }^{1}$ Department of Radiology, Graduate School of Medicine and Pharmaceutical Science, University of Toyama, 2630 Sugitani, Toyama 930-0194, Japan.

*corresponding. yamaken@med.u-toyama.ac.jp

\begin{abstract}
Information regarding the histological types of non-small cell lung cancer is essential to determine the treatment strategy. Although several radiomics studies using almost similar feature variables were reported, a considerable variation in the performances has been observed. In this study, as novel radiomic features, 2D Gabor filtering Minkowski functionals were used. They were calculated in rotational invariant and both scale and rotational invariant ways using circular shift operations of Gabor filters on nonenhanced computed tomographic images. Eighty-six patients (47 adenocarcinomas, 39 squamous cell carcinomas) were analyzed. Two independent observers manually delineated a single slice segmentation of a
\end{abstract}


tumor. Feature selection was made by neighborhood component analysis. Among various classifiers, 1-nearest neighbor gave a promising performance. The observer-averaged accuracy of rotational invariant analysis was $86.28 \%$ and that of both scale and rotational invariant one was $88.27 \%$. However, there was no common feature among the ten top-ranked features of each observer with the identical Gabor filtering type. Hence further study of the robustness is necessary to create a more reliable model.

\section{Introduction}

Accurate histologic classification of non-small cell lung cancer (NSCLC) between adenocarcinoma (AD) and squamous cell carcinoma $(\mathrm{SCC})$ is mandatory in selecting the best systemic therapeutic option for patients. The classification is not always feasible technically because of limited tissue samples of lung cancer ${ }^{1}$. A computed tomographic (CT) scan is a commonly exploited medical imaging tool for lung cancer patients. However, on the CT images, the visual differentiation between lung cancer histological subtypes is difficult. Radiomics is an objective approach to extract quantitative imaging features and perform the classification using features selected from the high-dimensional feature space. Several studies have been done, but the reported performances varied considerably (AUC 0.5 $0.893)^{2-9}$ 
This study aims to develop a novel radiomics method for the histological classification of NSCLC on nonenhanced CT images. 2D Gabor filtering (GF) Minkowski functionals (MFs) were used as quantitative imaging features. In the previous studies, a vast range of radiomics features in which MFs were not included have been investigated. MFs are fundamental quantities of Integral-geometry morphological analysis ${ }^{10}$. In 2D, area, perimeter, and Euler number characterize the geometrical and topological pattern formed by black-and-white (objects and background) binary images. MFs have proven to be very useful for statistical analysis of microstructures in material science ${ }^{11}$. They have also been used to detect tumor response heterogeneity to treatment and differentiate pseudoprogression from glioblastoma progression on MR images ${ }^{12-13}$. In the previous studies ${ }^{2-9}$, the majority of essential features were wavelet decomposed features. It implied that a single resolution approach was not sufficient and a multiresolution approach was useful. GF is one of the most commonly used methods to extract features at different scales and directions ${ }^{14}$. Unlike the discrete wavelet decomposition, GF can select the filtering direction arbitrarily and perform multi-directional analysis. Moreover, GF circular shift operation enables the analysis of object's rotational invariant (RI) and both scale and rotational invariant (SRI) properties ${ }^{15-16}$.

In radiomics study, a proper feature selection method improves the performance of machine learning ${ }^{17}$. Feature selection is a technique eliminating irrelevant and redundant 
features from high-dimensional data. Several nearest neighbor (NN)-based feature selection methods have been successfully developed. Wu et al. ${ }^{4}$ showed that reliefF, a popular NNbased method, was optimal among 24 feature selection methods for the histological classification of NSCLC. As a novel NN-based feature selection method, neighborhood component analysis (NCA) was proposed ${ }^{18-19}$. The better performance of NCA than that of the state-of-the-art NN-based methods was also reported ${ }^{19}$. In the present study, NCA was used as the feature selection method and the performances of various machine learning models were evaluated.

\section{Methods}

The radiomics analysis was performed using MATLAB (Release 2020a, The MathWorks, Natick, MA).

\section{Patients}

Ethics Committee, University of Toyama approved this study and waived informed consents.

This study was performed in accordance with the relevant guidelines and regulations. Radiation oncologist searched the clinical and radiological database of the institution. The data of NSCLC patients who received radiation therapy at the University of Toyama Hospital 
from April 2014 to May 2020 was collected. The inclusion criteria were as follows:(1) pathologically confirmed $A D$ and SCC except for favor $A D$ and SCC; (2) available nonenhanced CT images with a voxel size $0.39 \times 0.39 \times 1 \mathrm{~mm}^{3}$ before chemotherapy or radiotherapy. The patients with only ground-grass opacity (GGO) lesions were excluded. Definitive diagnosis was confirmed by cytology, biopsy, or surgical resection. Voxel size resampling was not used in this study, since the resampling was not sufficient for some intensity histogram and texture features ${ }^{20}$. The numerical values of those features were highly correlated with a voxel count of a tumor and feature normalization using the voxel count was proposed $^{21}$.

Eighty-six patients (47 AD and $39 \mathrm{SCC}$ ) were included. Table 1 summarizes the characteristics of patients. AD had a significantly higher female rate $\left(p=0.003, \chi^{2}\right.$ test). In T2 and T4 stage, there were significant differences between AD and SCC $(p=0.0006$, and $p=$ $0.002, \chi^{2}$ test). SCC had a significantly higher rate of $\mathrm{T} 3$ or more tumor than $\mathrm{AD}(p=0.0004$, $\chi^{2}$ test), while AD had a significantly higher rate of $\mathrm{T} 2$ or less tumor than $\operatorname{SCC}(p=0.0009$, $\chi^{2}$ test).

Table 1. Patient characteristics.

$$
\text { Variable } \quad \operatorname{AD}(n=47) \quad \operatorname{SCC}(n=39)
$$




\begin{tabular}{|c|c|c|}
\hline \multicolumn{3}{|c|}{ Age(years) } \\
\hline Median & 69 & 71 \\
\hline Range & $47-90$ & $46-85$ \\
\hline \multicolumn{3}{|l|}{ Gender } \\
\hline Male & 22 & 33 \\
\hline Female & 25 & 6 \\
\hline \multicolumn{3}{|l|}{ T-stage } \\
\hline $\mathrm{T} 1$ & 15(31.9\%) & $7(17.9 \%)$ \\
\hline $\mathrm{T} 2$ & $20(42.6 \%)$ & $2(5.1 \%)$ \\
\hline T3 & $6(12.8 \%)$ & $11(28.2 \%)$ \\
\hline T4 & $6(12.8 \%)$ & 19(48.7\%) \\
\hline
\end{tabular}

\section{Image acquisition}

One of four Multidetector CT systems (SOMATOM Definition AS+,SOMATOM Force, sensation cardiac 64, sensation 16, Siemens Medical Solutions, Forchheim, Germany) and one of five convolution kernels (170f/2, Br64d/2, Br69d/2, B65f, B75f) were used. CT scanning parameters were as follows: tube voltage of $120 \mathrm{kVp}$, tube current-time product of 47-329 $\mathrm{mAs}$, matrix size $512 \times 512$, pixel length $0.39 \mathrm{~mm}$, and slice thickness $1 \mathrm{~mm}$. 


\section{Region of interest (ROI) segmentation}

A tumor was segmented manually using image segmentation software (ITK-SNAP, version 3.6.0). Two independent observers did the segmentation as radiation oncologist (Obs. 1) and radiologist (Obs. 2). We delineated a single slice ROI of a tumor instead of 3D whole tumor segmentation because SCC had a significantly higher rate of advanced T-stage tumor than AD. Obs. 1 selected the slice so that the difference between voxel counts of AD and SCC becomes small. Obs. 2 selected the segmented slice arbitrarily. The following regions were not included in ROI: (1) separated or narrowly connected region; (2) GGO region; (3) invasive region out of lung field area; (4) non-tumor region like vessels, cavity and calcification to the extent possible. As seen in Supplementary Fig. S1, each observer selected a different slice for the same tumor and excluded the separated or narrowly connected regions.

\section{Gabor filter}

A two dimensional Gabor function $g(x, y)$ can be written as:

$$
g(x, y)=\frac{1}{2 \pi \sigma^{2}} \exp \left(-\frac{x^{\prime 2}+\gamma^{2} y^{\prime 2}}{2 \sigma^{2}}\right) \cos \left(\frac{2 \pi x^{\prime}}{u}\right)
$$

,where $x^{\prime}=x \cos \theta+y \sin \theta, \quad y^{\prime}=-x \sin \theta+y \cos \theta . \quad \sigma$ and $u$ are the standard deviations of the Gaussian factor of the Gabor function and preferred wavelength, 
respectively. $\theta$ specifies the orientation normal to the parallel stripes of a Gabor function. Aspect ratio $\gamma$ specifies the ellipticity of the Gabor function's support. Theyvalue of 0.5 was used. The half-response spatial frequency bandwidth (in octaves) of a Gabor filter was set to 1 , in which case, $\sigma$ and $u$ are connected as $\sigma=0.56 u$. The wavelength $u_{m}$ was logarithmically spaced as:

$$
u_{m}=a^{m} u_{\min }, m=\{0,1,2,3\}
$$

where minimum wavelength $u_{\min }=2$ and the scaling factor $a=2$, respectively.

Four orientations

$$
\theta_{n}=\frac{n \pi}{4}, \quad n=\{0,1,2,3\}
$$

were adopted. Sixteen Gabor filters $g_{m, n}(x, y)$ were applied to the original CT image $I(x, y)$.

The convolution gives GF images:

$$
G_{m, n}(x, y)=\sum_{s} \sum_{t} I(x, y) g_{m, n}(x-s, y-t)
$$

which represents a $4 \times 4$ matrix for each pixel location $(x, y)$. For each pixel location $(x, y)$ we found the position $\left(m_{\max }, n_{\max }\right)$ at which the matrix $G_{m, n}$ became maximum. Using circular shift operations of $m$ as $m_{\max } \rightarrow 0$ (if $m_{\max }=2$, then $\{0,1,2,3\} \rightarrow\{2,3,0,1\}$ ) to study scale invariant properties of an object and those of $n$ as $n_{\max } \rightarrow 0$ to study rotational invariant ones ${ }^{15-16}$, GF images were transformed to the rotational invariant (RI) and both scale and rotational invariant (SRI) forms as: 


$$
G_{m, n}(x, y) \rightarrow G_{m, n}^{R I}(x, y) \text { and } G_{m, n}^{S R I}(x, y)
$$

\section{Minkowski functionals}

To remove the highly noisy data effects, $1 \%$ of the data were saturated at low and high intensities. The intensities of segmented tumor images were linearly remapped onto uniform interval 0 to 1 . The simplest method of reducing gray-scale images to two-valued images (objects and background) is to use a threshold that is any number between 0 and 1 . Integralgeometry morphological image analysis consists of the calculation of MFs as a function of the threshold. Larkin et al. ${ }^{12}$ reported that thresholding steps of more than ten did not improve the classification performance. We also adopted ten thresholding steps from 0 to 1 with 0.1 intervals $(l=0,1, \ldots, 10)$ to obtain 11 binary images. In Supplementary Fig. S2 and S3, the representative examples of $\mathrm{ROI}$ on the original image, GF image and each thresholding image were shown. As seen in Supplementary Fig. S3, GF emphasizes the edge region of ROI.

In 2D, three MFs were calculated on each binary image: area $\left(A_{l}\right)$, perimeter $\left(U_{l}\right)$, and Euler number $\left(\chi_{l}\right)$. Euler number is the number of connected components minus the number of holes. MFs on GF image has the additional indices as $A_{l, m, n}$, where $m, n$ represents the indices of $G_{m, n}$. Following Larkin et al. ${ }^{12}$, the area and perimeter were normalized to remove 
any dependence on the voxel count of the tumor as

$$
A_{l . m, n} \rightarrow A_{l . m, n} / A_{0, m, n} \quad, \quad U_{l, m, n} \rightarrow
$$

$U_{l . m, n} /\left(A_{0 . m, n}\right)^{1 / 2}$

The schematic illustration for the process of MFs computation is shown in Fig. 1.

\section{Neighborhood Component Analysis}

The weighted distance between two d-dimensional feature vectors $\boldsymbol{x}_{i}$ and $\boldsymbol{x}_{j}$ (the corresponding class labels $y_{i}$ and $y_{j}$ ) of two samples is defined as:

$$
D_{w}\left(\boldsymbol{x}_{i}, \boldsymbol{x}_{j}\right)=\sum_{l=1}^{d} w_{l}^{2}\left|x_{i l}-x_{j l}\right|
$$

where $w_{l}$ is a weight associated with $l$ th feature. The probability of $\boldsymbol{x}_{i}$ selects $\boldsymbol{x}_{j}$ as its reference point is defined as:

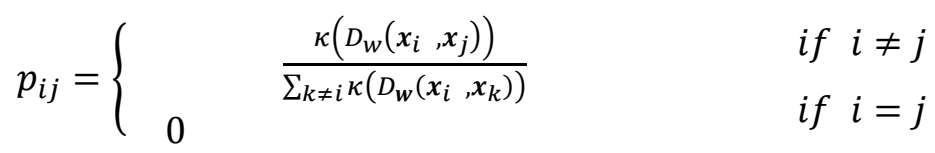

(8)

where $\kappa(z)=\exp (-z / \sigma)$ is a kernel function. The $\sigma$ value of 1 was used. The approximate leave-one-out cross-validation (CV) classification accuracy with a regularization term introduced to alleviate overfitting becomes as:

$$
\xi(\boldsymbol{w})=\sum_{i} \sum_{j} y_{i j} p_{i j}-
$$

$\lambda \sum_{l=1}^{d} w_{l}^{2}$

where $y_{i j}=1$ if $y_{i}=y_{j}$ and $y_{i j}=0$ otherwise. $\lambda>0$ is a regularization parameter. The 
objective function (9) is differentiable with respect to $w$, and used to optimize the feature weights by gradient ascent method. In this study, the regularization parameter $\lambda$ was tuned in the vicinity of $1 / n(n=86)$ to minimize the mean squared error (MSE) of leave-one-out CV. The features with weights of more than 0.5 were selected, although the threshold value was not optimized.

\section{Performance evaluation}

The classification performances in the original image, $G_{m, n}^{R I}$ and $G_{m, n}^{S R I}$ were evaluated using 10 -fold CV 10 times. The optimal classification model among various machine learning models was searched using the MATLAB classification learner app. It enable us to classify our data using various supervised machine learning algorithms and automatically compare the results in the same environment. The classification models available are: decision tree, discriminant analysis, Support Vector Machine, naïve Bayes model, logistic regression, nearest neighbors, and ensemble classification.

\section{Data availability}

The datasets generated and analyzed during this study are available from the corresponding author on a reasonable request. 


\section{Results}

\section{Segmentation}

The voxel counts of each observer for AD and SCC are shown in Fig. 2. Obs. 2 delineated ROI for an arbitrarily selected slice of a tumor. It is natural that SCC has a significantly larger voxel count than $A D$ in Obs. 2, because SCC has a higher rate of advanced T-stage tumor than AD. In contrast, Obs. 1 selected the slice so that the differences between voxel counts of $A D$ and SCC become small. As a result, there was no significant difference between the voxel counts of $A D$ and SCC.

\section{Minkowski functionals}

Figure 3 shows the area, perimeter, and Euler number calculated on the original image and $G_{0,0}^{S R I}$, respectively. GF MFs began to change at a smaller threshold than those of the original images. As the threshold increases, the increase of holes can increase the perimeter and decrease the Euler number. The increase in isolated regions increases the Euler number. At higher thresholds, the area and perimeter decrease as the number of remaining pixels decreases. 


\section{Neighborhood Component Analysis}

Before NCA, each feature's values were standardized to have a zero mean and a standard deviation of one to avoid bias caused by the difference of the value range of each feature. The features with a zero standard deviation were excluded. As shown in Fig. 4, MSE was very sensitive to $\lambda$, the careful fine-tuning of $\lambda$ was required to minimize MSE. The selected features using NCA are summarized in Table 2. There was no common feature among the ten top-ranked features of each observer with the identical GF type.

Table 2. 10 top-ranked features of each GF and observer using NCA.

\begin{tabular}{|c|c|c|c|c|}
\hline Observer & GF & $\lambda$ & $\begin{array}{c}\text { Number of } \\
\text { selected features }\end{array}$ & 10 top-ranked features \\
\hline & & & & in descending order \\
\hline 1 & None & 0.01140 & 5 & $U_{8}, U_{7}, U_{10}, U_{0}, A_{8}$ \\
\hline \multirow[t]{2}{*}{1} & $\mathrm{RI}$ & 0.01173 & 25 & $A_{1,1,3}, \quad U_{1,0,0}, \quad A_{1,3,3}, \quad \chi_{4,3,0}, \chi_{3,2,0}$ \\
\hline & & & & $\chi_{1,1,3}, \chi_{3,1,0}, \chi_{1,0,1}, U_{2,1,0}, \chi_{1,0,0}$ \\
\hline \multirow[t]{2}{*}{1} & SRI & 0.01141 & 22 & $\chi_{1,0,1}, U_{4,1,1}, U_{2,0,1}, A_{6,0,3}, U_{9,1,2}$ \\
\hline & & & & $\chi_{10,2,1}, \chi_{2,0,0}, A_{1,3,2}, U_{4,1,0}, U_{5,0,0}$ \\
\hline 2 & None & 0.01160 & 4 & $\chi_{1}, U_{1}, A_{9}, U_{6}$ \\
\hline 2 & $\mathrm{RI}$ & 0.01147 & 13 & $A_{4,2,0}, A_{1,0,0}, U_{1,0,3}, \chi_{3,3,3}, A_{1,3,2}$, \\
\hline
\end{tabular}




$A_{9,2,1}, A_{2,3,2}, U_{1,1,3}, \chi_{1,0,3}, \chi_{10,2,0}$
$U_{1,3,2}, \chi_{10,2,0}, \chi_{10,3,2}, A_{9,2,0}, U_{1,0,2}$,
$\chi_{3,0,3}, A_{6,1,2}, A_{1,1,3}, U_{1,3,0}, \chi_{3,0,2}$

\section{Performance Evaluation}

Among various machine learning models in MATLAB classification learner app, 1-NN with Manhattan distance measure (MD) and Euclidean distance measure (ED) performed most favorably. The classification performance of 1-NN are summarized in Table 3. Both types of GF improved the classification performance substantially and gave approximately the same observer-averaged performance.

Table 3. Classification performance of 1-NN (10-fold CV 10 times)

\begin{tabular}{cccccc}
\hline \multirow{2}{*}{ Observer } & GF & \multicolumn{2}{c}{ Accuracy $(\%)($ mean \pm s.d. $)$} & \multicolumn{2}{c}{ AUC (mean \pm s.d.) } \\
\cline { 3 - 6 } & & MD & ED & MD & ED \\
\hline 1 & None & $68.01 \pm 2.90$ & $67.20 \pm 2.37$ & $0.678 \pm 0.028$ & $0.667 \pm 0.025$ \\
1 & RI & $88.82 \pm 1.37$ & $89.54 \pm 1.91$ & $0.885 \pm 0.014$ & $0.896 \pm 0.021$ \\
1 & SRI & $86.17 \pm 2.53$ & $84.18 \pm 1.64$ & $0.861 \pm 0.022$ & $0.838 \pm 0.018$ \\
\hline 2 & None & $71.76 \pm 1.82$ & $74.09 \pm 1.44$ & $0.716 \pm 0.018$ & $0.742 \pm 0.015$ \\
2 & RI & $83.73 \pm 1.62$ & $83.72 \pm 1.54$ & $0.839 \pm 0.015$ & $0.841 \pm 0.014$
\end{tabular}




\begin{tabular}{cccccc}
2 & SRI & $90.36 \pm 2.33$ & $88.24 \pm 2.60$ & $0.902 \pm 0.025$ & $0.882 \pm 0.026$ \\
\hline Average & None & $69.89 \pm 2.35$ & $70.65 \pm 1.91$ & $0.697 \pm 0.023$ & $0.705 \pm 0.020$ \\
& & & & & \\
Average & RI & $86.28 \pm 1.50$ & $86.63 \pm 1.73$ & $0.862 \pm 0.014$ & $0.869 \pm 0.018$ \\
& & & & & \\
Average & SRI & $88.27 \pm 2.43$ & $86.21 \pm 2.12$ & $0.882 \pm 0.023$ & $0.860 \pm 0.022$ \\
\hline
\end{tabular}

\section{Discussion}

The present novel radiomic method for the histological classification of NSCLC provided the promising classification performance (accuracy about $88 \%$ and AUC 0.88 ) of 10 -fold CV. Basu et al. ${ }^{2}$ reported about $68 \%$ accuracy of 10 -fold CV for 74 tumors (AD 38, SCC 36). However, the performance should be evaluated using the independent test dataset preferably. Zhu et al. ${ }^{7}$ reported the best performance as AUC 0.893 using the training datasets $(n=81)$ and the independent test datasets $(n=48)$. Bashir et al. ${ }^{9}$ investigated a radiomic-based and semantic feature-based model using a training data set $(n=106)$ and an independent test data set $(n=100)$. On training data, the radiomics-based and semantic feature-based model gave $A \cup C=1$ and 0.78 , respectively, while on the test data, the semantic feature-based model outperformed the radiomics-based model $(A \cup C=0.82$ and 0.5$)$. Thus, the reported results differ considerably; therefore reproducibility of the results and generalizability to new independent data must be verified to create a reliable radiomics-based model. 
NN-based feature selection methods are unable to detect redundant features. Therefore, the correlation-based feature elimination before the feature selection stage was performed ${ }^{4}$. As another approach, the two-stage feature selection method combining minimal redundancy-maximal-relevance criterion (mRMR) and other feature selection method was proposed $^{22-23}$. In addition to NCA alone, we performed the two-stage method combining mRMR and NCA, but the performance weakened considerably in Obs. 2 (see Supplementary Fig. S4, S5 and Table S1).

The stability of extracted features must be evaluated in radiomics study. Several studies estimated the interobserver stabilities of various features by intra-class correlation coefficient ${ }^{24-26}$. MFs were not included in those studies. In the brain tumor analysis using MFs on T2-weighted MR images, MFs showed good interobserver agreement in Bland-Altman analysis $^{13}$. Although those methods are applied to normally distributed variables, the normality of many features in the present study was not confirmed by Jarque-Bera test. Hence, a nonparametric method was required. Aerts et al. ${ }^{27}$ used Friedman test, which is a nonparametric repeated measurement test. Haga et al. ${ }^{6}$ introduced an interobservervariability-based feature selection filter that selects features common to each observer. In this study, no common feature was found among ten top-ranked features of each observer. It might be because, in Obs. 2, the voxel count of SCC was significantly higher than that of AD. 
To remove any feature's dependence on the voxel count of ROI, feature normalization for the area and perimeter was performed as equation (6), but it might not be enough to remove that effect. A dataset without significant difference in T-stage between histological types is required to investigate further about the robustness.

\section{Conclusion}

We proposed the novel radiomics method using 2D RI and SRI GF MFs as the radiomics features and NCA as the feature selection method for histological classification of NSCLC on nonenhanced CT images. The promising results (about $88 \%$ accuracy) was obtained, although a more detailed study about the robustness is required.

\section{References}

1. Ho, C., Tong, K. M., Ramsden, K., lonescu, D. N. \& Laskin, J. Histologic classification of non-small-cell lung cancer over time: reducing the rates of not-otherwise-specified.

Curr. Oncol. 22, e164-e170; http://dx.doi.org/10.3747//co.22.2339 (2015).

2. Basu, S. et al. Developing a Classifier Model for Lung Tumors in CT-scan Images. IEEE International Conference on Systems, Man, and Cybernetics. 1306-1312 (2011).

3. Parmer, C. et al. Radiomic feature clusters and Prognostic Signatures specific for Lung and 
Head \& Neck cancer. Sci Rep 5, 11044; https://doi.org/10.1038/srep11044 (2015).

4. Wu, W. et al. Exploratory Study to Identify Radiomics Classifiers to identify radiomics Classifiers for Lung Cancer Histology. Front. Oncol. 6, 71; https://doi.org/10.3389/fonc.2016.00071 (2016).

5. Patil, R., Mahadevaiah, G. \& Dekker, A. An Approach Toward Automatic Classification of Tumor Histopathology of Non-Small Cell Lung Cancer Based on Radiomic Features. TOMOGRAPHY.ORG. 2, 374-377 (2016).

6. Haga, A. et al. Classification of early stage non-small cell lung cancers on computed tomographic images into histological types using radiomic features: interobserver delineation variability analysis. Radiological Physics and Technology 11, 27-35 (2018).

7. Zhu, X. et al. Radiomic signature as a diagnostic factor for histologic subtype classification of non-small cell lung cancer. Eur. Radiol. 28, 2772-2778 (2018).

8. Linning, E. et al. Radiomics for classification of lung cancer histological subtypes based on nonenhanced computed tomography. Acad. Radiol. 26, 1245-1252 (2019).

9. Bashir, U. et al. Non-invasive classification of non-small cell lung cancer: a comparison between random forest models utilizing radiomic and semantic features. Br. J. Radiol. 92 , 20190159; https://doi.org/10.1259/bjr.20190159 (2019). 
10. Michielsen, K. F. L. \& de Raedt, H. A. Integral-geometry morphological image analysis. Physics Reports-Review Section of Physics Letters. 347, 462-538 (2001).

11. Ohser, J. \& Mücklich, F. Statistical Analysis of Microstructures in Material Science. (ed. Barnett, V.) 107-140 (John Wiley \& Sons, 2000).

12. Larkin, T. J. et al. Analysis of Image Heterogeneity Using 2D Minkowski Functionals Dtects Tumor Responses to Treatment. Magn. Reson. Med. 71, 402-410 (2014).

13. Booth, T. C. et al. Analysis of heterogeneity in $T_{2}$-weighted MR images can differentiate pseudoprogression from progression in glioblastoma. PLOS ONE12, e0176528; https ://doi.org/10.1371/journal.pone.0176528 (2017).

14. Jain, A. K. \& Farrokhnia, F. Unsupervised Texture Segmentation Using Gabor Filters. Pattern Recognition. 24, 1167-1186 (1991)

15. Rahman, M. H., Pickering, M. R. \& Frater, M. R. Scale and Rotation Invariant Gabor Features for Texture Retrieval. IEEE International Conference on Digital Image Computing: Techniques and Applications. 602-607 (2011).

16. Kyrki, V., Kamarainen, J. K. \& Kälviäinen, H. Simple Gabor feature space for invariant object recognition. Pattern Recognition Letters. 25, 311-318 (2004).

17. Guyon, I. An Introduction to Variable and Feature Selection. Journal of Machine Learnng Reasrch. 3, 1157-1182 (2003). 
18. Goldberger, J., Hinton, G. E., Roweis, S. \& Salakhutdinov, R. R. Neighborhood Component Analysis. Advances in Neural Information Processing Systems 17, 13-18 (2004).

19. Yang, W., Wang, K. \& Zuo, W. Neighborhood Component Feature Selection for HighDimensional Data. Journal of Computers. 7, 161-168 (2012).

20. Shafiq-ul-Hassan, M. et al. Intrinsic dependencies of CT radiomic features on voxel size and number of gray levels. Medical Physics 44, 050-1062 (2017).

21. Shafiq-ul-Hassan, M. et al. Voxel size and gray level normalization of CT radiomics features in lung cancer. Sci. Rep. 8, 10545; https://doi.org/10.1038/s41598-018-28895-9 (2018).

22. Peng, H., Long, F. \& Ding, C. Feature Selection Based on Mutual Information: Criteria of Max-Dependency, Max-Relevance, and Min-Redundancy. IEEE TRANSACTIONONS ON PATTERN ANALYSIS AND MACHINE INTELLIGENCE. 27, 1226-1238 (2005).

23. Zhang, Y., Ding, C. \& Li, T. Gene selection algorithm by combining reliefF and mRMR. BMC Genomics. 9(Suppl 2),S27; https://doi.org/10.1186/1471-2164-9-S2-S27 (2008).

24. Griethuysen, J. et al. Computational Radiomics System to Decode the Radiographic Phenotype. Cancer Res. 77, e104-e107; https://doi.org/10.1158/0008-5472.CAN-17-0339 (2017). 
25. Parmer, C. et al. Robust Radiomics Feature Quantification Using Semiautomatic Volumetric Segmentation. PLOS ONE 9, e102107; https://doi.org/10.1371/journal.pone.01 $\underline{02107}$ (2014).

26. Owens, C. A. et al. Lung tumor segmentation methods: Impact on the uncertainty of radiomics features for non-small cell lung cancer. PLOS ONE 13, e0205003; https://d oi.org/10.1371/journal.pone.0205003 (2018).

27. Aerts, H. J. W. L. et al. Decoding tumour phenotype by noninvasive imaging using a quantitative radiomics approach. Nat. Commun. 5, 5006; https://doi.org/10.1038/nco$\underline{\mathrm{mms5006}}$ (2014).

\section{Acknowledgments}

The author would like to thank Mati Ur Rehman, Ph.D., Department of Radiology for English proofreading of this manuscript.

\section{Author contributions}

K.Y. conceived the idea, analyzed the data, and wrote the paper. N.N. provided image data manipulation and expert opinion. K.N. provided critical review and expert opinion. T.M. and J.S. provided expert opinion. All authors have reviewed the manuscript. 
Additional Information

Supplementary information accompanies this paper.

Competing interests

The authors declare no competing interests. 
Figures

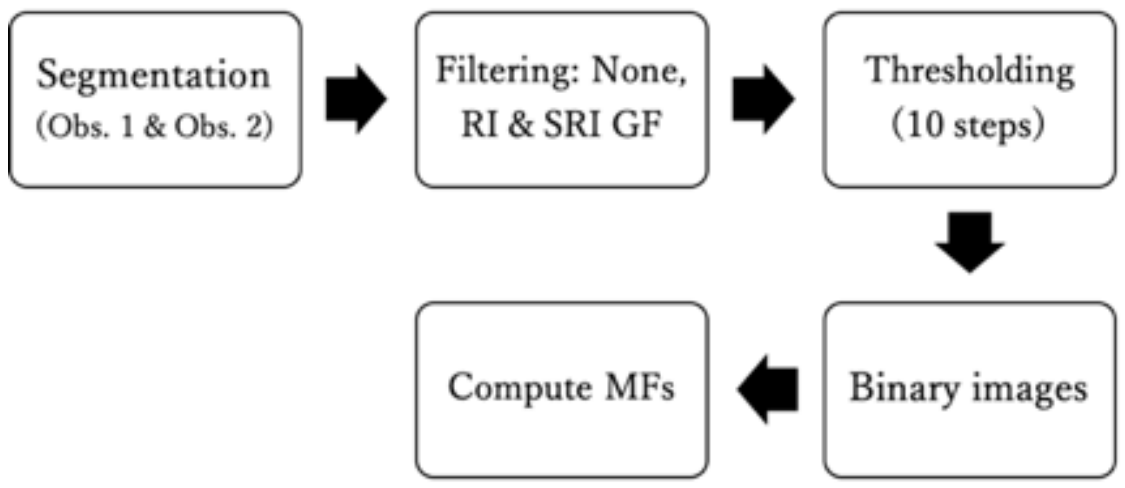

\section{Figure 1}

Schematic illustration for the process of MFs computation.

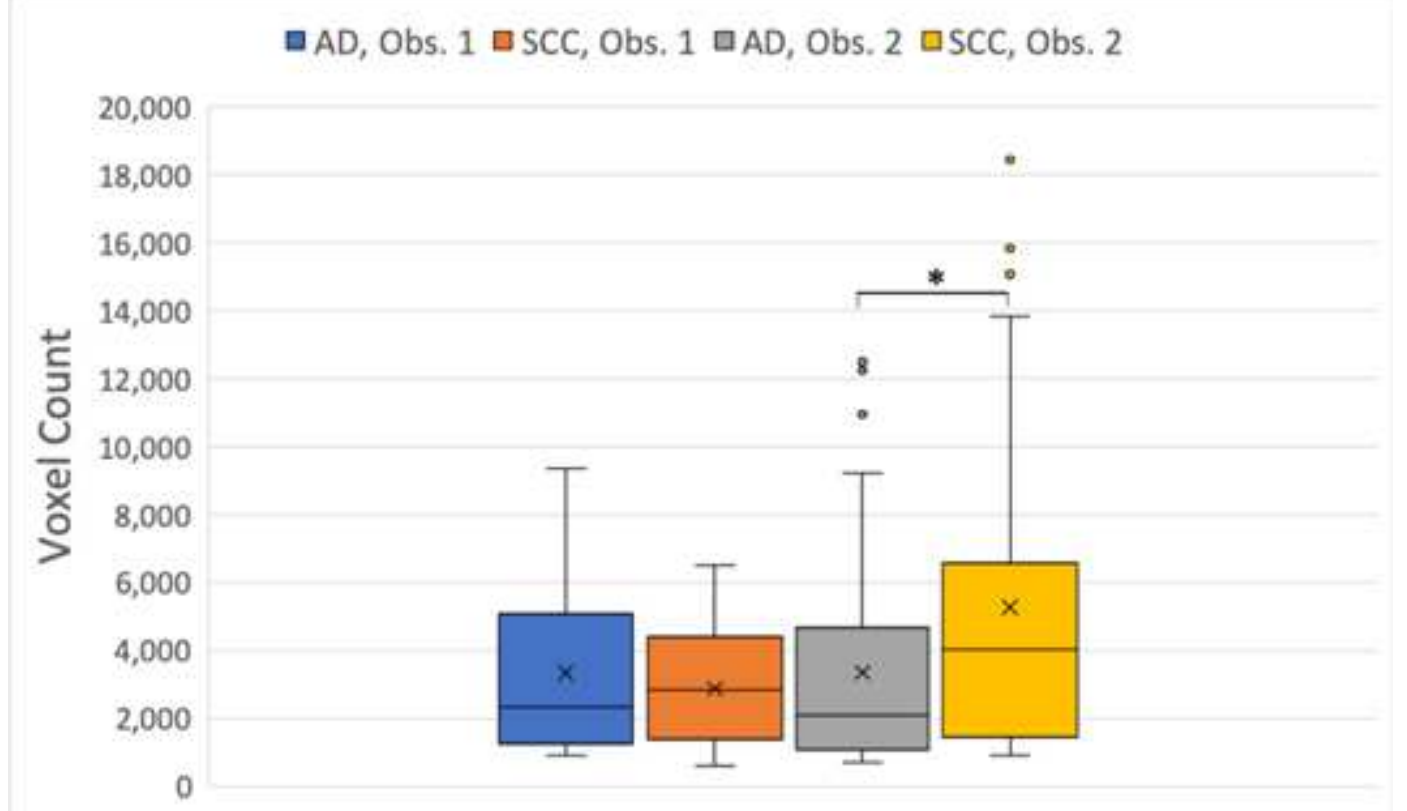

Figure 2

ROI voxel count of each histological type and observer. *: $p=0.045$, Kruskal Wallis and Dunn-Bonferroni test. 

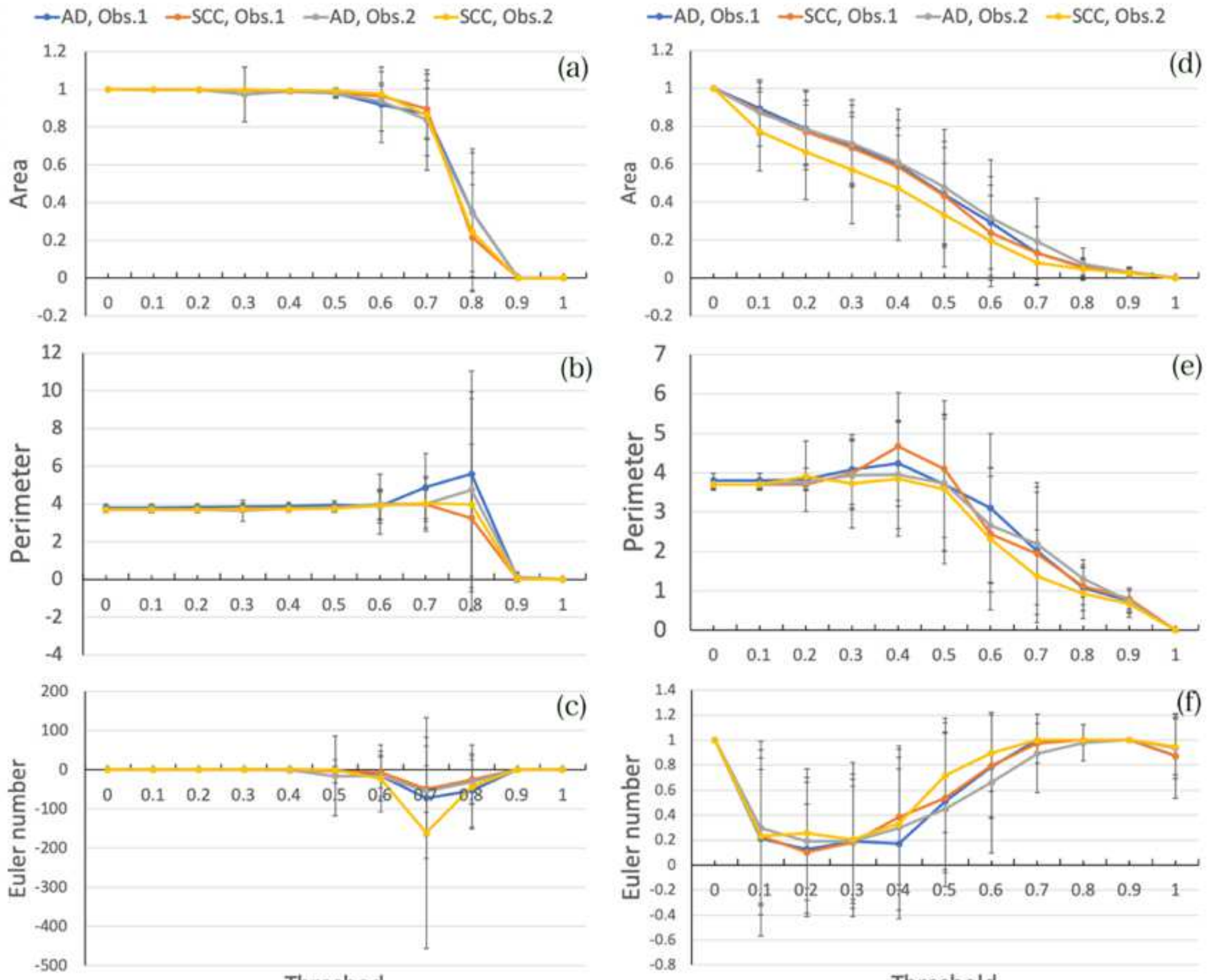

Threshod

Threshold

\section{Figure 3}

MFs(mean \pm s.d.) vs. threshold for each histological type and observer. Area, (b) Perimeter, and (c) Euler number on the original images. (d) Area, (e) Perimeter, and (f) Euler number on G_0,0^SRI. 

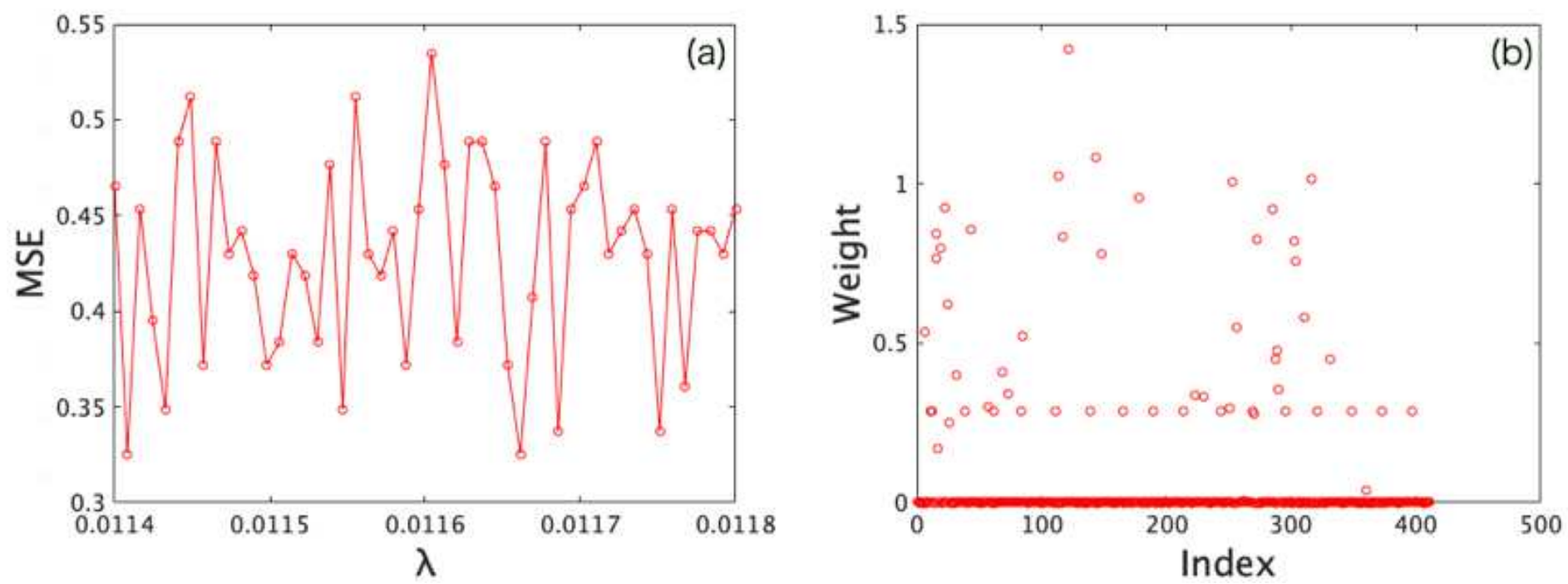

Figure 4

NCA for SRI GF and Obs.1. (a) MSE vs. $\lambda$ in the vicinity of $1 / n(n=86)$. The minimum MSE was 0.3256 at $\lambda=0.01141$. (b) Feature weights calculated by NCA with the tuned $\lambda$. Twenty-two features had the weights of more than 0.5 and were selected.

\section{Supplementary Files}

This is a list of supplementary files associated with this preprint. Click to download.

- SupplementaryInformation.docx 